\title{
OP-0193 Prevalence and Clinical Characteristics of Diabetes Mellitus in Lebanon: a National Survey
}

Ibrahim R. Bou-Orm, MD, MPH'1,2 ; Salim M. Adib, MD, DrPH ${ }^{1}$

${ }^{1}$ Faculty of Health Sciences - American University of Beirut; ${ }^{2}$ Faculty of Medicine - Saint-Joseph University of Beirut

\section{INTRODUCTION}

- Diabetes Mellitus (DM) in all its subtypes has been rapidly increasing worldwide, with particularly higher peaks in some Arab countries.

- This national study aimed to assess the prevalence and clinical aspects of DM in the Middle-Eastern country of Lebanon.

\section{METHODS}

- A national multi-stage, random household sample survey was conducted, using face-to-face interviews with one questionnaire per household.

- A total of 4500 households were selected from all areas based on a pre-existing sampling frame of the Lebanese population.

- Within selected households, 17,832 persons were accounted.

\section{RESULTS}

Table 1. Characteristics of surveyed households $(N=4500)$

\section{Variables}

Mean number of residents

Households with only one person

$3.96(\mathrm{SD}=1.77)$

$278(6.2 \%)$

Range of household membership

$1-15$

Geographic distribution ( $n, \%)$

Greater Beirut

$1320(29.3)$

Mount-Lebanon

$950(21.1)$

North-Lebanon

$920(20.4)$

South-Lebanon

$750(16.7)$

Bekaa

$560(12.5)$

\section{Household prevalence of diseases}

Diabetes

$1313(29.2 \%)$

Hypertension

$1406(31.2 \%)$

Hypercholesterolemia

$1131(25.1 \%)$

* Higher crowding indicates lower socio-economic status

Table 2. Socio-demographic characteristics of the sample and disease prevalence rates $(N=17,832)$

\section{Variables}

\begin{tabular}{|c|c|}
\hline Mean age in years (SD) & $35.9(20.4)$ \\
\hline \multicolumn{2}{|l|}{ Gender (n, \%) } \\
\hline Male & $8,937(50.1)$ \\
\hline Female & $8,895(49.9)$ \\
\hline \multicolumn{2}{|l|}{ Geographic distribution (n, \%) } \\
\hline Greater Beirut (urban/suburban) & $4,844(27.2)$ \\
\hline Elsewhere (small towns/rural) & $12,988(72.8)$ \\
\hline \multicolumn{2}{|c|}{ Prevalence of selected diseases $(95 \% \mathrm{Cl})$} \\
\hline Diabetes & $7.95(7.55-8.35)$ \\
\hline Hypertension & $9.03(8.61-9.45)$ \\
\hline Hypercholesterolemia & $7.37(6.99-7.75)$ \\
\hline
\end{tabular}

Table 3. Socio-demographic characteristics of persons with diabetes $(\mathrm{N}=1418)$

\begin{tabular}{|lc|}
\hline Variables & $\mathbf{n}(\%)$ \\
\hline Mean age in years (SD) & $60.3(13.0)$ \\
Age $\leq 40$ years & $6.7 \%$ \\
\hline Gender $(\mathbf{n}, \%)$ & \\
Male & $786(55.4)$ \\
Female & $632(44.6)$ \\
\hline Mean age at diagnosis (SD) & $49.4(15.9)$ \\
Age $\leq 25$ years & $63(4.5 \%)$ \\
Age $\leq 10$ years & $17(1.2 \%)$ \\
\hline
\end{tabular}

Figure 1. Prevalence of diabetes by age groups in Lebanon (2016) $(\mathrm{N}=17,832)$

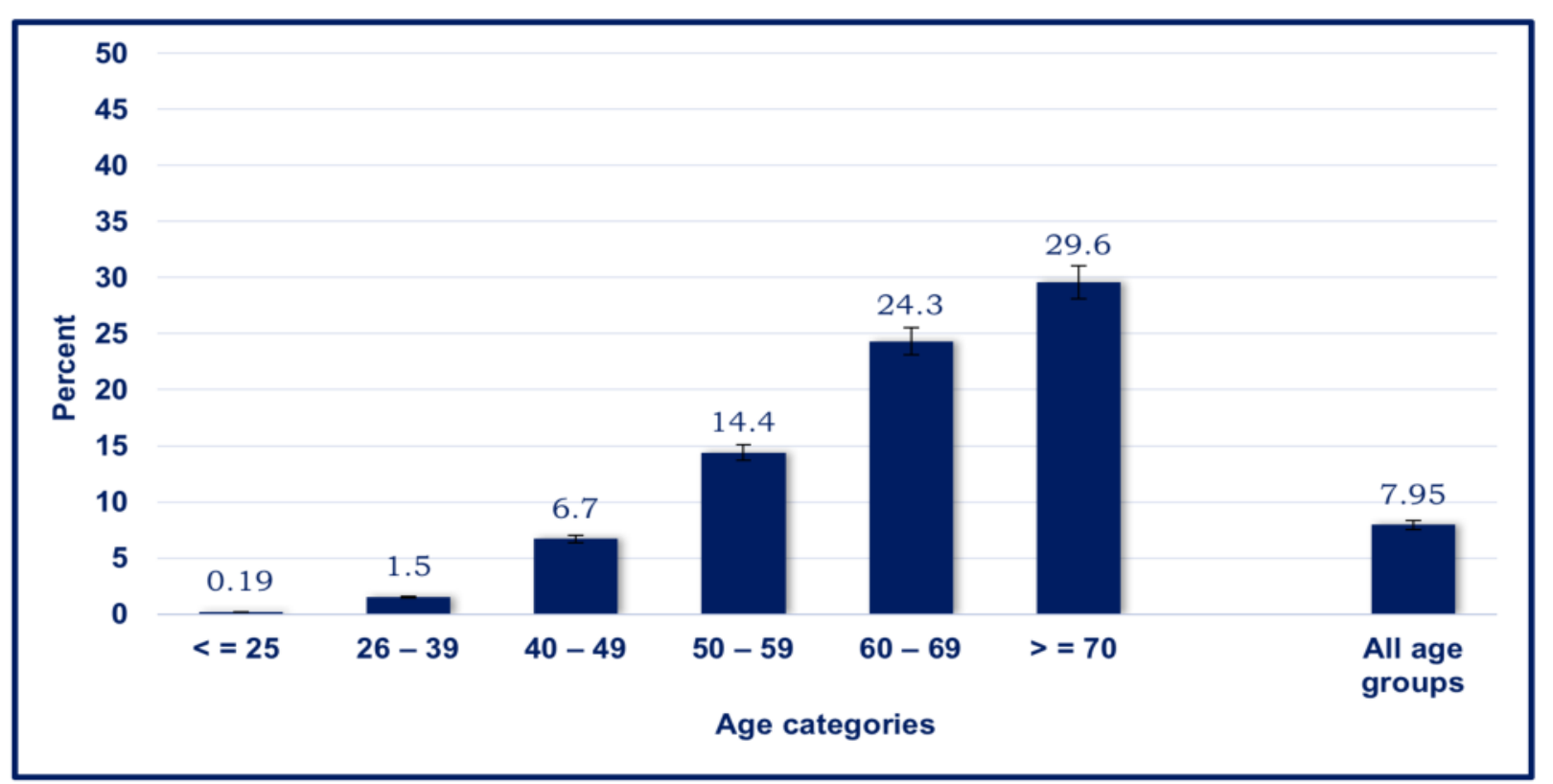

Table 4. Clinical characteristics of diabetes cases in Lebanon $(\mathrm{N}=1418)$

\begin{tabular}{|lc|}
\hline Variables & $\%$ \\
\hline Current use of Oral Anti- Diabetic drugs & $91 \%$ \\
\hline Current use of Insulin injections & $18 \%$ \\
\hline Last HbA1c during the previous 6 months & $76 \%$ \\
\hline Hypoglycemia requiring medical care & $12 \%$ \\
\hline Prevalence of complications & $23 \%$ \\
\hline
\end{tabular}

Figure 2. Prevalence of complications among people with diabetes $(\mathrm{N}=1418)$

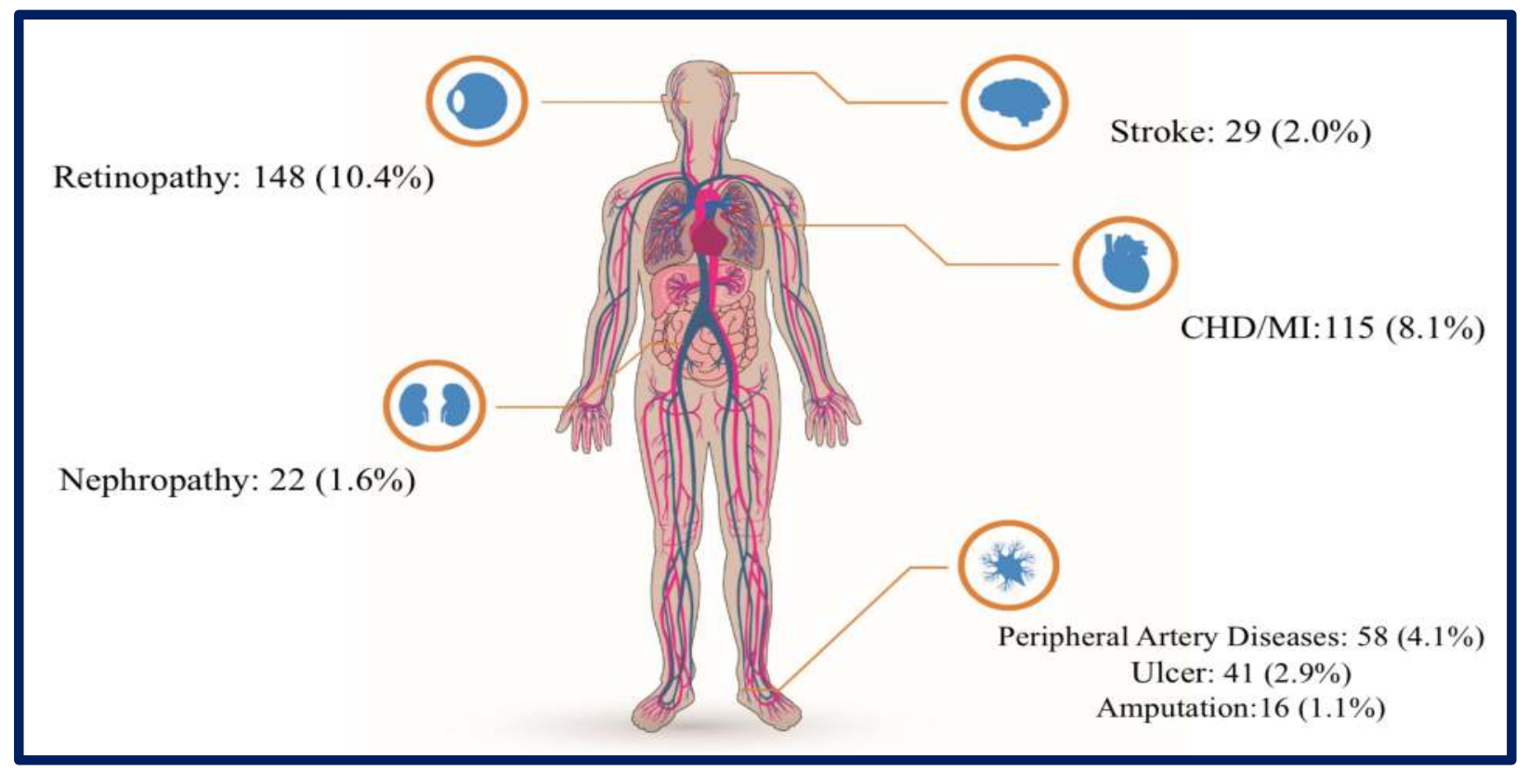

\section{CONCLUSIONS}

- Diabetes management appears to be deficient in Lebanon based on delays in standard control testing, hypoglycemic episodes and diabetes-related complications.

- Diabetic care should be devolved to PHC physicians as "gate-keepers" coordinating with specialists and keeping track of follow-up. 